\title{
Clinical Assessment of Micro-residual Tumors during Stereotactic Body Radiation Therapy for Hepatocellular Carcinoma
}

\author{
KENJI UEMOTO ${ }^{1,2}$, HIROSHI DOI ${ }^{1,3}$, HIROYA SHIOMI ${ }^{1}$, KOICHI YAMADA $^{1}$, DAISAKU TATSUMI ${ }^{1}$, \\ TAKU YASUMOTO ${ }^{1}$, MASAAKI TAKASHINA ${ }^{4}$, MASAHIKO KOIZUMI ${ }^{2}$ and RYOONG-JIN OH ${ }^{1}$ \\ ${ }^{1}$ Miyakojima IGRT Clinic, Osaka, Japan; \\ ${ }^{2}$ Division of Health Sciences, Osaka University Graduate School of Medicine and Health Science, Suita, Japan; \\ ${ }^{3}$ Department of Radiation Oncology, Kindai University of Medicine, Osaka, Japan; \\ ${ }^{4}$ Osaka Heavy Ion Therapy Center, Osaka, Japan
}

\begin{abstract}
Background: This study aimed to assess the need to consider microscopic invasion in terms of treatment planning in stereotactic body radiation therapy (SBRT) for hepatocellular carcinoma and elucidate the appropriate clinical target volume (CTV) margin. Patients and Methods: A total of 121 patients (with 146 liver tumors) who underwent SBRT between July 2007 and August 2016 were analyzed, regarding overall survival and local control $(L C)$. Results: The 2- and 5-year LC rates were $91.5 \%$ and $89.8 \%$, respectively. Planning target volume (PTV) margin $<8 \mathrm{~mm}$ was associated with poor LC. Of the 77 patients with PTV margin of $<8 \mathrm{~mm}$, age $<75$ years was associated with poor $L C$, while alpha-fetoprotein (AFP) $\leq 20 \mathrm{ng} / \mathrm{ml}$ was associated with good LC. Conclusion: In patients with high AFP levels, there is a possibility of microscopic invasion around the tumor, suggesting that LC may be improved by adding an additional clinical target volume margin to the gross tumor volume.
\end{abstract}

Hepatocellular carcinoma (HCC) is the most common primary hepatic tumor, developing in approximately $90 \%$ of patients with advanced cirrhosis (1). As local treatment for HCC, surgical resection and radiofrequency ablation (RFA) therapy have been established; however, these interventions are often complicated by certain factors, such as the liver reserve capacity, localization of the tumor, and degree of

Correspondence to: Kenji Uemoto, M. Sc., Miyakojima IGRT Clinic, 1-16-22, Miyakojima-ku, Osaka, 534-0021, Japan. Tel: +81 669233501, Fax: +81 669233520, e-mail: uemoto@osaka-igrt.or.jp

Key Words: Stereotactic body radiation therapy, intensity-modulated radiotherapy, stereotactic ablative radiation therapy, hepatocellular carcinoma. progression $(2,3)$. Promising results have been achieved using stereotactic body radiation therapy (SBRT), which allows for administration of high radiation doses to the primary tumor, while sparing the normal liver tissue (4-10). Therefore, the use of radical radiotherapy for unresectable HCC has increased dramatically in recent years (11-20). In addition, the excellent outcomes observed after liver SBRT have recently been reported to be comparable with those of RFA treatment (21).

Consequently, as the prescribed radiation doses are escalated, and particularly the high fractional doses associated with SBRT, it is becoming increasingly important to improve the accuracy of the target volume definition. In addition, due to the more accurate organ specification provided by image-guided radiation therapy (IGRT), the planning target volume (PTV) margin can now be optimized, with a minimization of patient set-up uncertainties.

Advanced radiation therapy techniques, including IGRT, tumor-tracking, and respiratory gating, may allow for a reduced PTV margin for SBRT. Furthermore, modern imaging techniques enable a more precise delineation of the gross tumor volume (GTV). However, none of the available imaging techniques can currently robustly identify the clinical target volume (CTV). Furthermore, there is a potential danger associated with IGRT techniques in terms of creating a false sense of confidence regarding margin reduction around the CTV (22). In addition, under normal circumstances, a CTV margin would not be added to the GTV when using SBRT (23). However, it remains poorly understood whether a CTV margin is actually necessary.

With this in mind, the purpose of the present study was to elucidate the optimal CTV margin from clinical data of patients receiving SBRT for HCC. 


\section{Patients and Methods}

Patient selection. The present study was conducted according to the principles of the Declaration of Helsinki. The Institutional Review Board of Miyakojima IGRT Clinic (Osaka, Japan) approved the data collection and analysis (approval no. 9), with written informed consent for radiotherapy being obtained from all patients. The HCC diagnosis was established based on the results of imaging studies using computed tomography $(\mathrm{CT})$ or magnetic resonance imaging (MRI), since pathological confirmation was not feasible for SBRT candidates. The exclusion criteria were as follows: (i) patients with insufficient blood collection data before SBRT; (ii) patients whose follow-up was discontinued within 12 months after SBRT; and (iii) patients who had manually reduced PTV margins to protect the gastrointestinal tract. As a result, a total of 146 liver tumors from 121 patients who received SBRT at Miyakojima IGRT Clinic between July 2007 and August 2016 were analyzed. The patient characteristics are shown in Table I. Between July 2007 and April 2013, we treated 69 tumors using a PTV that was created by adding an 8-mm uniform expansion to the internal target volume (ITV) in all directions. However, based on the clinical results and an evaluation of tumor position reproducibility by IGRT techniques, from May 2013 onward, for 77 tumors, the PTV margin was generated by adding a 4- or 6-mm uniform expansion to the ITV (4$\mathrm{mm}$ or $6-\mathrm{mm}$ expansion for tumors near and far from the vertebral bodies, respectively)

SBRT technique. SBRT for HCC treatment was performed as previously described (24). Briefly, CT scans for treatment planning were obtained using a 4 slice BrightSpeed Excel ${ }^{\mathrm{TM}}$ (GE Healthcare Bio Sciences Corp., Pittsburgh, PA, USA) scanner between July 2007 and June 2014, and a 64 slice SOMATOM Definition AS Open RT Pro edition (Siemens Healthcare, Munich, Germany) scanner from July 2014 onward. MRI images for treatment planning were obtained using a SIGNA EXCITE HDx 1.5-T (GE Healthcare Bio Sciences Corp.) MRI scanner. Contrast enhanced, 4 dimensional CT scans and gadolinium ethoxybenzyl diethylenetriamine pentaacetic acid based contrast-enhanced MRI images were used to determine the GTV. The CTV margin was not added to the GTV at our Institution. The ITV was defined as the superposition of all CTVs from the different respiratory phase data of the 4-dimensional CT. The PTV was created by adding a 4-, 6-, or $8 \mathrm{~mm}$ margin in all directions to the ITV. The prescribed radiation doses were documented at the reference point using conformal beams in 38 tumors, or were prescribed to deliver the tumor dose to $95 \%$ of the PTV using intensity-modulated radiation therapy in 108 tumors There was no statistically difference in a dose-volume histogram within the target volume, even with any irradiation technique. We administered a prescription dose equivalent to a biological effective dose 10 of approximately 80 Gy. Fractioned regimens were scheduled to spare organs at risks, including the normal liver tissue. SBRT was performed using a $6 \mathrm{MV}$ linear accelerator (Novalis, BrainLAB AG, Munich, Germany).

Follow-up. Local control (LC) was defined as the absence of local failure. The LC and overall survival (OS) times were defined as the intervals between the start of SBRT and the date of diagnosis of local failure or the date of death, respectively. Local failures were identified by experienced physicians using CT and MRI, and defined as any regrowth of the target tumor or the appearance of tumor staining in the target tumor on contrast enhanced images. Toxicity was evaluated using the Common Terminology Criteria for Adverse Events version 3.0 (25).

Statistical analysis. Quantitative variables are described as the median and range, and qualitative variables as percentages. The OS and LC were calculated using the Kaplan-Meier method, and statistical differences were evaluated using the log rank test. Cox proportional hazards models were used to evaluate the factors affecting the LC and OS. The results are reported as the hazard ratio (HR) and corresponding 95\% confidence interval (CI). Variables with $p$-values of less than 0.20 in the univariate analyses were analyzed in the multivariate models. Multivariate analyses were performed by Cox regression. All statistical analyses were performed using JMP software version 12.2.0 (SAS Institute, Cary, NC, USA). $p$-Values of less than 0.05 were considered statistically significant.

\section{Results}

Patient eligibility and tumor characteristics. Of the 121 eligible patients, 11 patients received SBRT for two different lesions simultaneously, 11 patients received SBRT for two different lesions that occurred sequentially, and one patient received SBRT for three different lesions that occurred sequentially. The median follow-up duration after SBRT for all patients was 21 months (range=2-70 months).

Local control. Considering all lesions, the LC rates were $91.5 \%$ and $89.8 \%$ at 2 and 5 years, respectively (Figure 1a). Multivariate analysis showed that a PTV margin $<8 \mathrm{~mm}$ $(\mathrm{HR}=13.04,95 \% \mathrm{CI}=1.18-143.70 p=0.036)$ was associated with poor LC (Table II). The 2-year LC rates were $97.8 \%$ and $88.5 \%$ in the $8-\mathrm{mm}$ and $<8-\mathrm{mm}$ margin groups, respectively $(p=0.034)$ (Figure $1 \mathrm{~b})$.

Survival. For patients overall, the OS rates were $66.8 \%$ and $43.7 \%$ at 2 and 5 years, respectively (Figure 2a). Multivariate analysis showed that a performance status of 0 was associated with good $\mathrm{OS}(\mathrm{HR}=0.47495 \% \mathrm{CI}=0.246-$ $0.914 p=0.026$ ), while a previous history of undergoing transarterial chemoembolization was associated with poor OS $(\mathrm{HR}=2.520,95 \% \mathrm{CI}=1.202-5.283 p=0.014)$ (Table III). The 2-year OS rates were $73.7 \%$ and $57.0 \%$ in the groups with performance status 0 and $\geq 1$, respectively $(p=0.052)$ (Figure 2b). The 2-year OS rates were $76.2 \%$ and $59.0 \%$ for patients with and without a history of transarterial chemoembolization, respectively $(p=0.037)$ (Figure $2 c)$.

Toxicity. In terms of toxicity, two patients (1.5\%) experienced cholangiectasis, one of which was grade 3 . Two patients $(1.5 \%)$ experienced grade 1 radiation pneumonitis, with both treated sites being segment 7 of the liver. One patient $(0.7 \%)$ experienced radiation mucositis. One patient $(0.7 \%)$ experienced a grade $1 \mathrm{rib}$ fracture, with this patient having been irradiated for a nearby lesion from the initial 
Table I. Patient and treatment characteristics (121 patients and 146 tumors).

\begin{tabular}{|c|c|c|c|c|}
\hline Characteristic & & Total & $\begin{array}{l}\text { PTV margin } 8 \mathrm{~mm} \\
\quad \text { (69 tumors) }\end{array}$ & $\begin{array}{l}\text { PTV margin }<8 \mathrm{~mm} \\
\quad \text { (77 tumors) }\end{array}$ \\
\hline \multirow[t]{2}{*}{ Gender, n (\%) } & Male & $70(57.9)$ & $36(60.0)$ & $34(55.7)$ \\
\hline & Female & $51(42.1)$ & $24(40.0)$ & $27(44.3)$ \\
\hline Age, years & Median (range) & $75(44-91)$ & $74(44-89)$ & $75(48-91)$ \\
\hline \multirow[t]{5}{*}{ Performance status, n (\%) } & 0 & $77(57.1)$ & $34(52.3)$ & $43(61.4)$ \\
\hline & 1 & $42(31.1)$ & $21(32.3)$ & $21(30.0)$ \\
\hline & 2 & $13(9.6)$ & $9(13.8)$ & $4(5.7)$ \\
\hline & 3 & $2(1.5)$ & $0(0.0)$ & $2(2.9)$ \\
\hline & 4 & $1(0.7)$ & $1(1.5)$ & $0(0.0)$ \\
\hline \multirow[t]{4}{*}{ AJCC TNM Stage, n (\%) } & I & $62(51.2)$ & $25(41.7)$ & $37(60.7)$ \\
\hline & II & $41(33.9)$ & $25(41.7)$ & $16(26.2)$ \\
\hline & III & $14(11.6)$ & $7(11.7)$ & $7(11.5)$ \\
\hline & IV & $4(3.3)$ & $3(5.0)$ & $1(1.6)$ \\
\hline \multirow[t]{3}{*}{ Etiology, n (\%) } & $\mathrm{HBV}$ & $22(17.7)$ & $6(10.0)$ & $16(25.0)$ \\
\hline & $\mathrm{HCV}$ & $71(57.3)$ & $39(65.0)$ & $32(50.0)$ \\
\hline & Other & $31(25.0)$ & $15(25.0)$ & $16(25.0)$ \\
\hline \multirow[t]{2}{*}{ Child-Pugh class, n (\%) } & A & $115(78.8)$ & $54(78.3)$ & $61(79.2)$ \\
\hline & $\mathrm{B}$ & $31(21.2)$ & $15(21.7)$ & $16(20.8)$ \\
\hline \multirow[t]{2}{*}{ Portal vein tumor thrombosis, $\mathrm{n}(\%)$} & Yes & $12(7.0)$ & $6(8.7)$ & $6(7.8)$ \\
\hline & No & $134(91.8)$ & $63(91.3)$ & $71(92.2)$ \\
\hline \multirow[t]{2}{*}{ Previous surgery, n (\%) } & Yes & $23(19.0)$ & $13(21.7)$ & $10(16.4)$ \\
\hline & No & $98(81.0)$ & $47(78.3)$ & $51(83.6)$ \\
\hline \multirow[t]{2}{*}{ Previous TACE, n (\%) } & Yes & $77(52.7)$ & $36(52.2)$ & $41(53.2)$ \\
\hline & No & $69(47.3)$ & $33(47.8)$ & $36(46.8)$ \\
\hline \multirow[t]{2}{*}{ Previous RFA, n (\%) } & Yes & $38(26.0)$ & $22(31.9)$ & $16(20.8)$ \\
\hline & No & $108(74.0)$ & $47(68.1)$ & $61(79.2)$ \\
\hline \multirow[t]{3}{*}{ Alpha-fetoprotein, $\mathrm{ng} / \mathrm{ml}$} & Median (range) & $14.3(1.4-46939.0)$ & $16.4(1.4-16558.9)$ & $11.4(2.2-46939.0)$ \\
\hline & $\leq 20$ & $79(58.5)$ & $36(55.4)$ & $43(61.4)$ \\
\hline & $>20$ & $56(41.5)$ & $29(44.6)$ & $27(38.6)$ \\
\hline \multirow[t]{3}{*}{ PIVKA-II, mAU/ml } & Median (range) & $52(7.0-75000.0)$ & $54(7.0-40315.0)$ & $47(10.0-75000.0)$ \\
\hline & $\leq 40$ & $59(43.7)$ & $27(41.5)$ & $32(45.7)$ \\
\hline & $>40$ & $76(56.3)$ & $38(58.5)$ & $38(54.3)$ \\
\hline \multirow[t]{3}{*}{ Platelet count, $10^{4} / \mu 1$} & Median (range) & $11.3(2.7-32.0)$ & $10.3(2.8-26.6)$ & $12.6(2.7-31.5)$ \\
\hline & $\leq 10$ & $55(40.7)$ & $30(46.2)$ & $25(35.7)$ \\
\hline & $>10$ & $80(59.3)$ & $35(53.8)$ & $45(64.3)$ \\
\hline GTV, cc & Median (range) & $7.8(0.3-257.0)$ & $8.6(0.3-257.0)$ & $6.5(0.3-109.0)$ \\
\hline \multirow[t]{3}{*}{ Size of maximum diameter, $\mathrm{mm}, \mathrm{n}(\%)$} & $\leq 30$ & $71(48.6)$ & $30(43.5)$ & $41(53.2)$ \\
\hline & $30-50$ & $47(32.2)$ & $28(40.6)$ & $19(24.7)$ \\
\hline & $>50$ & $28(19.2)$ & $11(15.9)$ & $17(22.1)$ \\
\hline ITV, cc & Median (range) & $12.5(0.7-257.0)$ & $11.7(1.4-257.0)$ & $13(0.7-151.0)$ \\
\hline PTV, cc & Median (range) & $45.3(4.3-523.0)$ & $51.6(16.2-523.0)$ & $34.8(4.3-252.0)$ \\
\hline \multirow[t]{2}{*}{ Irradiation technology, $\mathrm{n}(\%)$} & IMRT & $108(74.0)$ & $34(49.3)$ & $74(96.1)$ \\
\hline & $\mathrm{CB}$ & $38(26.0)$ & $35(50.7)$ & $3(3.9)$ \\
\hline Total dose, Gy & Median (range) & $45(30-64)$ & $45(30-56)$ & $45(40-64)$ \\
\hline Number of fractions & Median (range) & $5(4-20)$ & $5(4-16)$ & $5(4-20)$ \\
\hline Fraction size, Gy & Median (range) & $8.3(3.0-12.0)$ & $8(3.3-12.0)$ & $8.5(3.0-11.0)$ \\
\hline Total prescription BED10, Gy & Median (range) & $80(48.0-106.0)$ & $80(48.0-105.6)$ & $80(56.0-100.0)$ \\
\hline Liver volume, cc & Median (range) & $1026(419-1803)$ & $1081(419-1803)$ & $1005(568-1740)$ \\
\hline Mean dose to the liver, Gy & Median (range) & $18.1(4.6-53.9)$ & $19.9(9.6-53.9)$ & $17.4(4.6-47.8)$ \\
\hline
\end{tabular}

AJCC, American Joint Committee on Cancer; HBV, Hepatitis B virus; HCV, Hepatitis C virus; TACE, transarterial chemoembolization; RFA, radiofrequency ablation; PIVKA-II, protein induced by vitamin K absence/antagonist-II; GTV, gross tumor volume; ITV, internal target volume; PTV, planning target volume; IMRT, intensity-modulated radiation therapy; $\mathrm{CB}$, conformal beam; BED, biological effective dose.

treatment. Finally, 34 patients $(25.2 \%)$ experienced ascites, three (2.2\%) experienced jaundice, and two (1.5\%) experienced pleural effusion. Hematological toxicities were not observed in patients who exhibited hematological abnormalities prior to radiotherapy, with no apparent changes from baseline. 

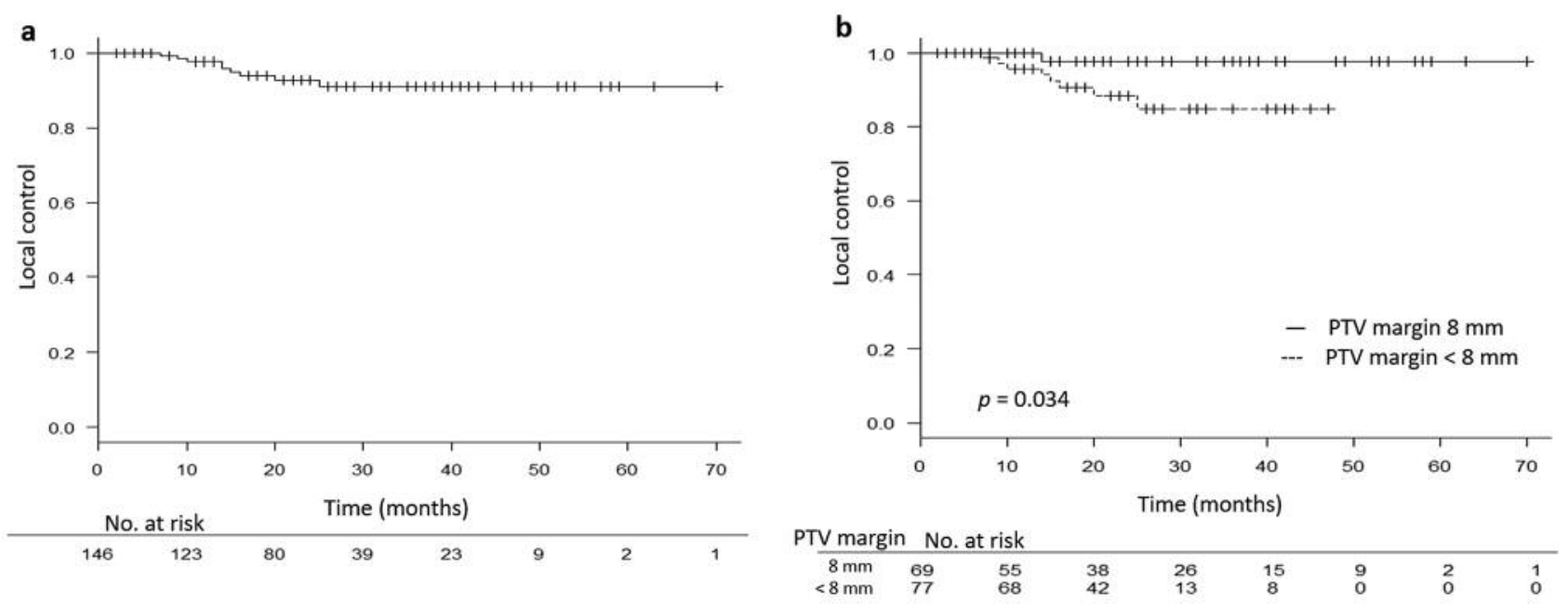

Figure 1. Local control rates from the time of stereotactic body radiotherapy treatment in all patients $(n=146)(a)$ and according to planning target volume margin $(b)$.

PTV margin. We further analyzed 77 patients who were treated using PTV margins of $<8 \mathrm{~mm}$. As a result, multivariate analysis showed that age $<75$ years was associated with poor $\mathrm{LC}(\mathrm{HR}=15.06,95 \% \mathrm{CI}=1.20-189.80$ $p=0.036$ ), while alpha-fetoprotein (AFP) $\leq 20 \mathrm{ng} / \mathrm{ml}$ was associated with good LC (HR=0.125, 0.021-0.758 $p=0.024)$ (Table IV). Among these patients, the 2-year LC rates were $96.0 \%$ and $80.3 \%$ in those aged $\geq 75$ years and $<75$ years, respectively $(p=0.013$ ) (Figure $3 \mathrm{a}$ ). The 2 -year LC rates were $97.8 \%$ and $69.2 \%$ in the groups with AFP $\leq 20 \mathrm{ng} / \mathrm{ml}$ and $>20 \mathrm{ng} / \mathrm{ml}$, respectively $(p=0.007)$ (Figure $3 b)$.

\section{Discussion}

As local therapy, surgical resection and RFA are wellestablished treatments for HCC. Several factors can make these interventions more challenging, including poor liver function, ill-defined anatomical barriers and vessels from ultrasound, and tumors with portal infiltration. Modern radiotherapy techniques, including SBRT, have recently attracted increasing attention for various malignancies, including as treatment with curative intent. Moreover, SBRT has recently been reported to be equally effective as RFA for HCC (21).

In contrast to these results, the recurrence of HCC tumors after SBRT is hard to predict since there are multiple, complex factors associated with recurrence and disease progression. Accordingly, in the present study, there were many variables significantly related to LC in the univariate analysis for all patients; however, the PTV margin was the only independent factor in the multivariate analysis. This result implies that reducing the PTV margin results in a higher risk of recurrence. Engels et al. reported that patients with prostate cancer who underwent radiotherapy with a reduced PTV margin, positioned with implanted markers, had poorer treatment outcomes than those without the implanted markers (5-year freedom from biochemical failure rate: $58 \%$ vs. $91 \%, p=0.02$ ). This result shows the potential danger associated with image guidance techniques creating a false sense of confidence regarding margin reduction around the CTV (22). Recurrence is another important treatment outcome that must be considered when reducing the PTV margin. Therefore, we examined a subgroup of 77 patients whose PTV margins were $<8 \mathrm{~mm}$. As a result, age and AFP levels were the only independent factors associated with LC in the multivariate analysis.

While modern imaging techniques can enable precise delineation of the GTV, none can robustly detect the microscopic extent of HCC. Moreover, although the CTV is defined as the volume that includes both gross and microscopic extensions of the malignancy, the CTVs used in SBRT are frequently equal to the GTV (23). At present, the necessity for a CTV margin remains under debate.

The extent of microscopic disease from liver tumors and the implications for radiotherapy are limited and remain unknown. In addition to direct invasion, HCC is also associated with a high risk of daughter nodules around the tumor tis sue, which can result in locoregional recurrence (26). Wang et al. reported a potential margin of microscopic disease beyond the gross tumor of $8.0 \mathrm{~mm}$; however, $94.7 \%$ of patients with HCC had microscopic extensions $\leq 3.5 \mathrm{~mm}$, with a median of $1 \mathrm{~mm}$ (27). In another report by the same group, 149 resected HCC specimens were found to have a mean diameter of $5.8 \mathrm{~cm}$ (range $=1.0-22.0 \mathrm{~cm}$ ), with microinvasion not observed in 
Table II. Factors associated with local control of 146 tumors.

\begin{tabular}{|c|c|c|c|c|c|c|}
\hline \multirow[t]{2}{*}{ Factor } & & \multirow[t]{2}{*}{$\mathrm{n}(\%)$} & \multicolumn{2}{|c|}{ Univariate analysis } & \multicolumn{2}{|c|}{ Multivariate analysis } \\
\hline & & & Hazard ratio $(95 \% \mathrm{CI})$ & $p$-Value & Hazard ratio $(95 \% \mathrm{CI})$ & $p$-Value \\
\hline \multirow[t]{2}{*}{ Gender } & Male & $87(59.6)$ & $0.964(0.259-3.594)$ & 0.956 & - & - \\
\hline & Female & $59(40.4)$ & 1 & & - & \\
\hline \multirow[t]{2}{*}{ Age, years } & $<75$ & $72(52.4)$ & $8.967(1.121-71.72)$ & 0.039 & $8.496(0.849-85.06)$ & 0.069 \\
\hline & $\geq 75$ & $74(47.6)$ & 1 & & 1 & \\
\hline \multirow[t]{2}{*}{ Performance status } & 0 & $82(56.2)$ & $0.740(0.199-2.758)$ & 0.654 & - & - \\
\hline & $\geq 1$ & $64(43.8)$ & 1 & & - & \\
\hline \multirow[t]{2}{*}{ AJCC TNM Stage } & I & $72(49.3)$ & $0.556(0.117-2.112)$ & 0.407 & - & - \\
\hline & $\geq \mathrm{II}$ & $74(50.7)$ & 1 & & - & \\
\hline \multirow[t]{4}{*}{ Etiology } & HBV & $23(15.8)$ & $2.909(0.727-11.65)$ & 0.131 & $2.969(0.510-17.28)$ & 0.226 \\
\hline & Other & $123(84.2)$ & 1 & & 1 & \\
\hline & $\mathrm{HCV}$ & $86(58.9)$ & $0.911(0.245-3.393)$ & 0.889 & - & - \\
\hline & Other & $60(41.1)$ & 1 & & - & \\
\hline \multirow[t]{2}{*}{ Child-Pugh class } & A & $115(78.8)$ & $0.859(0.178-4.137)$ & 0.850 & - & - \\
\hline & $\mathrm{B}$ & $31(21.2)$ & 1 & & - & \\
\hline \multirow[t]{2}{*}{ Portal vein tumor thrombosis } & Yes & $12(7.0)$ & $1.635(0.204-13.11)$ & 0.644 & - & - \\
\hline & No & $134(91.8)$ & 1 & & - & \\
\hline \multirow[t]{2}{*}{ Previous surgery } & Yes & $27(18.5)$ & $4,18 \mathrm{E}-08$ & 0.999 & - & - \\
\hline & No & $119(81.5)$ & 1 & & - & \\
\hline \multirow[t]{2}{*}{ Previous TACE } & Yes & $77(52.7)$ & $7.980(0.997-63.87)$ & 0.050 & $3.050(0.262-35.44)$ & 0.373 \\
\hline & No & $69(47.3)$ & 1 & & 1 & \\
\hline \multirow[t]{2}{*}{ Previous RFA } & Yes & $38(26.0)$ & $0.741(0.153-3.582)$ & 0.709 & - & - \\
\hline & No & $108(74.0)$ & 1 & & - & \\
\hline \multirow[t]{2}{*}{ Alpha-fetoprotein, $\mathrm{ng} / \mathrm{mL}$} & $\leq 20$ & $86(58.9)$ & $0.268(0.067-1.074)$ & 0.063 & $0.347(0.070-1.712)$ & 0.194 \\
\hline & $>20$ & $60(41.1)$ & 1 & & 1 & \\
\hline \multirow[t]{2}{*}{ PIVKA-II, mAU/mL } & $\leq 40$ & $63(43.2)$ & $1.369(0.367-5.107)$ & 0.640 & - & - \\
\hline & $>40$ & $83(56.8)$ & 1 & & - & \\
\hline \multirow{2}{*}{ Platelet count, $10^{4} / \mu 1$} & $\leq 10$ & $58(39.7)$ & $0.816(0.204-3.268)$ & 0.774 & - & - \\
\hline & $>10$ & $88(60.3)$ & 1 & & - & \\
\hline \multirow[t]{2}{*}{ GTV, cc } & $\leq 10$ & $82(56.2)$ & $0.654(0.175-2.445)$ & 0.528 & - & - \\
\hline & $>10$ & $64(43.8)$ & 1 & & - & \\
\hline \multirow{4}{*}{$\begin{array}{l}\text { Size of maximum } \\
\text { diameter, } \mathrm{mm}\end{array}$} & $\leq 30$ & $71(48.6)$ & $0.533(0.142-1.997)$ & 0.350 & - & - \\
\hline & $>30$ & $75(51.4)$ & 1 & & - & \\
\hline & $\leq 50$ & $118(80.8)$ & $0.605(0.125-2.930)$ & 0.532 & - & - \\
\hline & $>50$ & $28(19.2)$ & 1 & & - & \\
\hline PTV, cc & $\leq 40$ & $65(44.5)$ & $0.400(0.100-1.607)$ & 0.197 & $0.420(0.081-2.165)$ & 0.300 \\
\hline & $>40$ & $81(55.5)$ & 1 & & 1 & \\
\hline PTV margin, mm & $<8$ & $77(52.7)$ & $6.924(0.863-55.56)$ & 0.069 & $13.04(1.183-143.7)$ & 0.036 \\
\hline & 8 & $69(47.3)$ & 1 & & 1 & \\
\hline Irradiation technology & IMRT & $108(74.0)$ & 281300000 & 0.998 & - & - \\
\hline & $\mathrm{CB}$ & $38(26.0)$ & 1 & & - & \\
\hline Total dose, Gy & $<45$ & $64(43.8)$ & $0.142(0.018-1.134)$ & 0.066 & $0.692(0.018-26.61)$ & 0.843 \\
\hline & $\geq 45$ & $82(56.2)$ & 1 & & 1 & \\
\hline Number of fractions & $\leq 5$ & $81(55.5)$ & $0.165(0.034-0.795)$ & 0.025 & $0.1535(0.014-1.702)$ & 0.127 \\
\hline & $>5$ & $65(44.5)$ & 1 & & 1 & \\
\hline Fraction size, Gy & $<10$ & $96(65.8)$ & $5.268(0.658-42.19)$ & 0.118 & $0.811(0.020-32.13)$ & 0.911 \\
\hline & $\geq 10$ & $50(34.2)$ & 1 & & 1 & \\
\hline Total prescription BED10. Gy & $<80$ & $61(41.8)$ & $0.424(0.114-1.586)$ & 0.203 & - & - \\
\hline & $\geq 80$ & $85(58.2)$ & 1 & & - & \\
\hline Liver volume, cc & $\leq 1000$ & $65(44.5)$ & $0.562(0.140-2.252)$ & 0.416 & - & - \\
\hline & $>1000$ & $81(55.5)$ & 1 & & - & \\
\hline Mean dose to the liver, Gy & $<18$ & $68(46.6)$ & $3.458(0.717-16.68)$ & 0.122 & $4.736(0.686-32.68)$ & 0.115 \\
\hline & $\geq 18$ & $78(53.4)$ & 1 & & 1 & \\
\hline
\end{tabular}

CI, Confidence interval; AJCC, American Joint Committee on Cancer; HBV, Hepatitis B virus; HCV, Hepatitis C virus; TACE, transarterial chemoembolization; RFA, radiofrequency ablation; PIVKA-II, protein induced by vitamin K absence/antagonist-II; GTV, gross tumor volume; ITV, internal target volume; PTV, planning target volume; IMRT, intensity-modulated radiation therapy; CB, conformal beam; BED, biological effective dose. 
Table III. Factors associated with overall survival in 121 patients

\begin{tabular}{|c|c|c|c|c|c|c|}
\hline \multirow[t]{2}{*}{ Factor } & & \multirow[t]{2}{*}{$\mathrm{n}(\%)$} & \multicolumn{2}{|c|}{ Univariate analysis } & \multicolumn{2}{|c|}{ Multivariate analysis } \\
\hline & & & Hazard ratio $(95 \% \mathrm{CI})$ & $p$-Value & Hazard ratio $(95 \% \mathrm{CI})$ & $p$-Value \\
\hline \multirow[t]{2}{*}{ Gender } & Male & $70(57.9)$ & $0.927(0.525-1.640)$ & 0.795 & - & - \\
\hline & Female & $51(42.1)$ & 1 & & - & \\
\hline \multirow[t]{2}{*}{ Age, years } & $<75$ & $59(48.8)$ & $1.703(0.948-3.059)$ & 0.075 & $1.639(0.839-3.202)$ & 0.149 \\
\hline & $\geq 75$ & $62(51.2)$ & 1 & & 1 & \\
\hline \multirow[t]{2}{*}{ Performance status } & 0 & $72(59.5)$ & $0.577(0.328-1.017)$ & 0.057 & $0.474(0.246-0.914)$ & 0.026 \\
\hline & $\geq 1$ & $49(40.5)$ & 1 & & 1 & \\
\hline \multirow[t]{2}{*}{ AJCC TNM Stage } & I & $62(51.2)$ & $0.774(0.437-1.372)$ & 0.381 & - & - \\
\hline & $\geq \mathrm{II}$ & $59(48.8)$ & 1 & & - & \\
\hline \multirow[t]{4}{*}{ Etiology } & $\mathrm{HBV}$ & $22(18.2)$ & $1.075(0.502-2.302)$ & 0.853 & - & - \\
\hline & Other & $99(81.8)$ & 1 & & - & \\
\hline & $\mathrm{HCV}$ & $71(58.7)$ & $1.242(0.687-2.247)$ & 0.473 & - & - \\
\hline & Other & $50(41.3)$ & 1 & & - & \\
\hline \multirow[t]{2}{*}{ Child-Pugh class } & $\mathrm{A}$ & $95(78.5)$ & $0.537(0.294-0.979)$ & 0.042 & $0.699(0.300-1.631)$ & 0.408 \\
\hline & B & $26(21.5)$ & 1 & & 1 & \\
\hline \multirow[t]{2}{*}{ Portal vein tumor thrombosis } & Yes & $12(9.9)$ & $1.503(0.637-3.543)$ & 0.352 & - & - \\
\hline & No & $109(90.1)$ & 1 & & - & \\
\hline \multirow[t]{2}{*}{ Previous surgery } & Yes & $23(19.0)$ & $0.953(0.446-2.038)$ & 0.901 & - & - \\
\hline & No & $98(81.0)$ & 1 & & - & \\
\hline \multirow[t]{2}{*}{ Previous TACE } & Yes & $69(57.0)$ & $1.890(1.025-3.482)$ & 0.042 & $2.520(1.202-5.283)$ & 0.014 \\
\hline & No & $52(43.0)$ & 1 & & 1 & \\
\hline \multirow[t]{2}{*}{ Previous RFA } & Yes & $35(28.9)$ & $1.302(0.724-2.341)$ & 0.378 & - & - \\
\hline & No & $86(71.1)$ & 1 & & - & \\
\hline \multirow[t]{2}{*}{ Alpha-fetoprotein, $\mathrm{ng} / \mathrm{mL}$} & $\leq 20$ & $73(60.3)$ & $0.556(0.315-0.981)$ & 0.043 & $0.767(0.382-1.542)$ & 0.457 \\
\hline & $>20$ & $48(39.7)$ & 1 & & 1 & \\
\hline \multirow{2}{*}{ PIVKA-II, mAU/mL } & $\leq 40$ & $53(43.8)$ & $0.673(0.374-1.208)$ & 0.185 & $0.666(0.338-1.313)$ & 0.240 \\
\hline & $>40$ & $68(56.2)$ & 1 & & 1 & \\
\hline \multirow[t]{2}{*}{ Platelet count, $10^{4} / \mu \mathrm{L}$} & $\leq 10$ & $47(38.8)$ & $1.727(0.978-3.049)$ & 0.06 & $1.999(0.890-4.492)$ & 0.094 \\
\hline & $>10$ & $74(61.2)$ & 1 & & 1 & \\
\hline \multirow[t]{2}{*}{ GTV, cc } & $\leq 10$ & $64(52.9)$ & $0.443(0.249-0.790)$ & 0.006 & $0.311(0.044-2.203)$ & 0.242 \\
\hline & $>10$ & $57(47.1)$ & 1 & & 1 & \\
\hline \multirow{4}{*}{$\begin{array}{l}\text { Size of maximum } \\
\text { diameter, } \mathrm{mm}\end{array}$} & $\leq 30$ & $49(40.5)$ & $0.513(0.281-0.939)$ & 0.030 & $2.365(0.749-7.472)$ & 0.142 \\
\hline & $>30$ & $72(59.5)$ & 1 & & 1 & \\
\hline & $\leq 50$ & $92(76.0)$ & $0.656(0.346-1.243)$ & 0.196 & $1.788(0.722-4.428)$ & 0.209 \\
\hline & $>50$ & $29(24.0)$ & 1 & & 1 & \\
\hline PTV, cc & $\leq 40$ & $47(38.8)$ & $0.388(0.201-0.747)$ & 0.005 & $0.367(0.133-1.012)$ & 0.053 \\
\hline & $>40$ & $74(61.2)$ & 1 & & 1 & \\
\hline PTV margin, mm & $<8$ & $61(50.4)$ & $0.618(0.338-1.127)$ & 0.117 & $0.719(0.328-1.580)$ & 0.412 \\
\hline & 8 & $60(49.6)$ & 1 & & 1 & \\
\hline Irradiation technology & IMRT & $86(71.1)$ & $0.614(0.343-1.098)$ & 0.100 & $0.438(0.1811-1.057)$ & 0.066 \\
\hline & $\mathrm{CB}$ & $35(28.9)$ & 1 & & 1 & \\
\hline Total dose, Gy & $\leq 45$ & $50(41.3)$ & $0.764(0.427-1.365)$ & 0.363 & - & - \\
\hline & $>45$ & $71(58.7)$ & 1 & & - & \\
\hline Number of fractions & $\leq 5$ & $66(54.5)$ & $0.514(0.290-0.910)$ & 0.022 & $0.466(0.197-1.104)$ & 0.083 \\
\hline & $>5$ & $55(45.5)$ & 1 & & 1 & \\
\hline Fraction size, Gy & $<10$ & $82(67.8)$ & $2.440(1.212-4.910)$ & 0.012 & $1.815(0.610-5.401)$ & 0.327 \\
\hline & $\geq 10$ & $39(32.2)$ & 1 & & 1 & \\
\hline Total prescription BED10, Gy & $<80$ & $52(43.0)$ & $1.798(1.016-3.183)$ & 0.044 & $1.732(0.577-5.197)$ & 0.265 \\
\hline & $\geq 80$ & $69(57.0)$ & 1 & & 1 & \\
\hline Liver volume, $\mathrm{cc}$ & $\leq 1,000$ & $53(43.8)$ & $0.739(0.411-1.328)$ & 0.316 & - & - \\
\hline & $>1,000$ & $68(56.2)$ & 1 & & - & \\
\hline Mean dose to the liver, Gy & $<18$ & $56(46.3)$ & $0.800(0.452-1.416)$ & 0.444 & - & - \\
\hline & $\geq 18$ & $65(53.7)$ & 1 & & - & \\
\hline
\end{tabular}

CI, Confidence interval; AJCC, American Joint Committee on Cancer; HBV, Hepatitis B virus; HCV, Hepatitis C virus; TACE, transarterial chemoembolization; RFA, radiofrequency ablation; PIVKA-II, protein induced by vitamin K absence/antagonist-II; GTV, gross tumor volume; ITV, internal target volume; PTV, planning target volume; IMRT, intensity-modulated radiation therapy; CB, conformal beam; BED, biological effective dose. 
Table IV. Factors associated with local control in 77 patients treated using a planning target volume (PTV) margin $<8 \mathrm{~mm}$.

\begin{tabular}{|c|c|c|c|c|c|c|}
\hline \multirow[t]{2}{*}{ Factor } & & \multirow[t]{2}{*}{$\mathrm{n}(\%)$} & \multicolumn{2}{|c|}{ Univariate analysis } & \multicolumn{2}{|c|}{ Multivariate analysis } \\
\hline & & & Hazard ratio $(95 \% \mathrm{CI})$ & $p$-Value & Hazard ratio $(95 \% \mathrm{CI})$ & $p$-Value \\
\hline \multirow[t]{2}{*}{ Gender } & Male & $44(57.1)$ & $0.838(0.210-3.355)$ & 0.803 & - & - \\
\hline & Female & $33(42.9)$ & 1 & & - & \\
\hline \multirow[t]{2}{*}{ Age, years } & $<75$ & $37(48.1)$ & $8.894(1.093-72.35)$ & 0.041 & $15.06(1.195-189.8)$ & 0.036 \\
\hline & $\geq 75$ & $40(51.9)$ & 1 & & 1 & \\
\hline \multirow[t]{2}{*}{ Performance status } & 0 & $46(59.7)$ & $0.583(0.145-2.343)$ & 0.447 & - & - \\
\hline & $\geq 1$ & $31(40.3)$ & 1 & & - & \\
\hline \multirow{2}{*}{ AJCC TNM Stage } & I & $45(58.4)$ & $1.209(0.288-5.071)$ & 0.795 & - & - \\
\hline & $\geq \mathrm{II}$ & $32(41.6)$ & 1 & & - & \\
\hline \multirow[t]{4}{*}{ Etiology } & HBV & $16(20.8)$ & $2.880(0.685-12.11)$ & 0.149 & $2.652(0.437-16.09)$ & 0.289 \\
\hline & Other & $61(79.2)$ & 1 & & 1 & \\
\hline & $\mathrm{HCV}$ & $42(54.5)$ & $1.312(0.313-5.500)$ & 0.71 & - & - \\
\hline & Other & $35(45.5)$ & 1 & & - & \\
\hline \multirow[t]{2}{*}{ Child-Pugh class } & A & $61(79.2)$ & $1.683(0.207-13.71)$ & 0.627 & - & - \\
\hline & $\mathrm{B}$ & $16(20.8)$ & 1 & & - & \\
\hline \multirow{2}{*}{ Portal vein tumor thrombosis } & Yes & $6(7.8)$ & $2.194(0.268-17.96)$ & 0.464 & - & - \\
\hline & No & $71(92.2)$ & 1 & & - & \\
\hline \multirow[t]{2}{*}{ Previous surgery } & Yes & $11(14.3)$ & 1,181 & 0.998 & - & - \\
\hline & No & $66(85.7)$ & 1 & & - & \\
\hline \multirow[t]{2}{*}{ Previous TACE } & Yes & $41(53.2)$ & $7.860(0.963-64.14)$ & 0.054 & $3.643(0.225-59.04)$ & 0.363 \\
\hline & No & $36(46.8)$ & 1 & & 1 & \\
\hline \multirow[t]{2}{*}{ Previous RFA } & Yes & $16(20.8)$ & $1.104(0.221-5.520)$ & 0.904 & - & - \\
\hline & No & $61(79.2)$ & 1 & & - & \\
\hline \multirow[t]{2}{*}{ Alpha-fetoprotein, ng/mL } & $\leq 20$ & $46(59.7)$ & $0.146(0.029-0.732)$ & 0.019 & $0.125(0.021-0.758)$ & 0.024 \\
\hline & $>20$ & $31(40.3)$ & 1 & & 1 & \\
\hline \multirow{2}{*}{ PIVKA-II, mAU/mL } & $\leq 40$ & $34(44.2)$ & $1.226(0.307-4.906)$ & 0.773 & - & - \\
\hline & $>40$ & $43(55.8)$ & 1 & & - & \\
\hline \multirow[t]{2}{*}{ Platelet count, $10^{4} / \mu \mathrm{L}$} & $\leq 10$ & $26(33.8)$ & $0.581(0.117-2.886)$ & 0.506 & - & - \\
\hline & $>10$ & $51(66.2)$ & 1 & & - & \\
\hline \multirow[t]{2}{*}{ GTV, cc } & $\leq 10$ & $44(57.1)$ & $0.547(0.136-2.197)$ & 0.395 & - & - \\
\hline & $>10$ & $33(42.9)$ & 1 & & - & \\
\hline \multirow{4}{*}{$\begin{array}{l}\text { Size of maximum } \\
\text { diameter, } \mathrm{mm}\end{array}$} & $\leq 30$ & $41(53.2)$ & $0.589(0.146-2.380)$ & 0.458 & - & - \\
\hline & $>30$ & $36(46.8)$ & 1 & & - & \\
\hline & $\leq 50$ & $60(77.9)$ & $0.588(0.118-2.929)$ & 0.517 & - & - \\
\hline & $>50$ & $17(22.1)$ & 1 & & - & \\
\hline \multirow[t]{2}{*}{ PTV, cc } & $\leq 40$ & $41(53.2)$ & $0.380(0.090-1.599)$ & 0.187 & $0.690(0.089-5.349)$ & 0.723 \\
\hline & $>40$ & $36(46.8)$ & 1 & & 1 & \\
\hline \multirow[t]{2}{*}{ Total dose, Gy } & $<45$ & $32(41.6)$ & $0.139(0.017-1.136)$ & 0.066 & $0.770(0.037-16.07)$ & 0.866 \\
\hline & $\geq 45$ & $45(58.4)$ & 1 & & 1 & \\
\hline Number of fractions & $\leq 5$ & $41(53.2)$ & $0.175(0.035-0.876)$ & 0.034 & $0.121(0.007-1.983)$ & 0.139 \\
\hline & $>5$ & $36(46.8)$ & 1 & & 1 & \\
\hline Fraction size, Gy & $<10$ & $52(67.5)$ & $4.421(0.543-36.02)$ & 0.165 & $1.218(0.052-28.82)$ & 0.903 \\
\hline & $\geq 10$ & $25(32.5)$ & 1 & & 1 & \\
\hline Total prescription BED10. Gy & $<80$ & $29(37.7)$ & $1.047(0.248-4.418)$ & 0.950 & - & - \\
\hline & $\geq 80$ & $48(62.3)$ & 1 & & - & \\
\hline Liver volume, cc & $\leq 1000$ & $38(49.4)$ & $0.460(0.108-1.966)$ & 0.295 & - & - \\
\hline & $>1000$ & $39(50.6)$ & 1 & & - & \\
\hline Mean dose to the liver, Gy & $<18$ & $43(55.8)$ & $2.485(0.498-12.41)$ & 0.267 & - & - \\
\hline & $\geq 18$ & $34(44.2)$ & 1 & & - & \\
\hline
\end{tabular}

CI, Confidence interval; AJCC, American Joint Committee on Cancer; HBV, Hepatitis B virus; HCV, Hepatitis C virus; TACE, transarterial chemoembolization; RFA, radiofrequency ablation; PIVKA-II, protein induced by vitamin K absence/antagonist-II; GTV, gross tumor volume; ITV, internal target volume; IMRT, intensity-modulated radiation therapy; BED, biological effective dose.

$47.0 \%$ of patients (28). Moreover, microinvasion distances $\leq 2$ $\mathrm{mm}$ were found in $94.5 \%$ of patients with AFP levels $<400$ $\mu \mathrm{g} / \mathrm{l}$. AFP is an important predictor of prognosis and recurrence (29-32). Many authors have reported AFP to be an independent predictor of poor prognosis, even in patients receiving curative resection (33-36). These findings were 

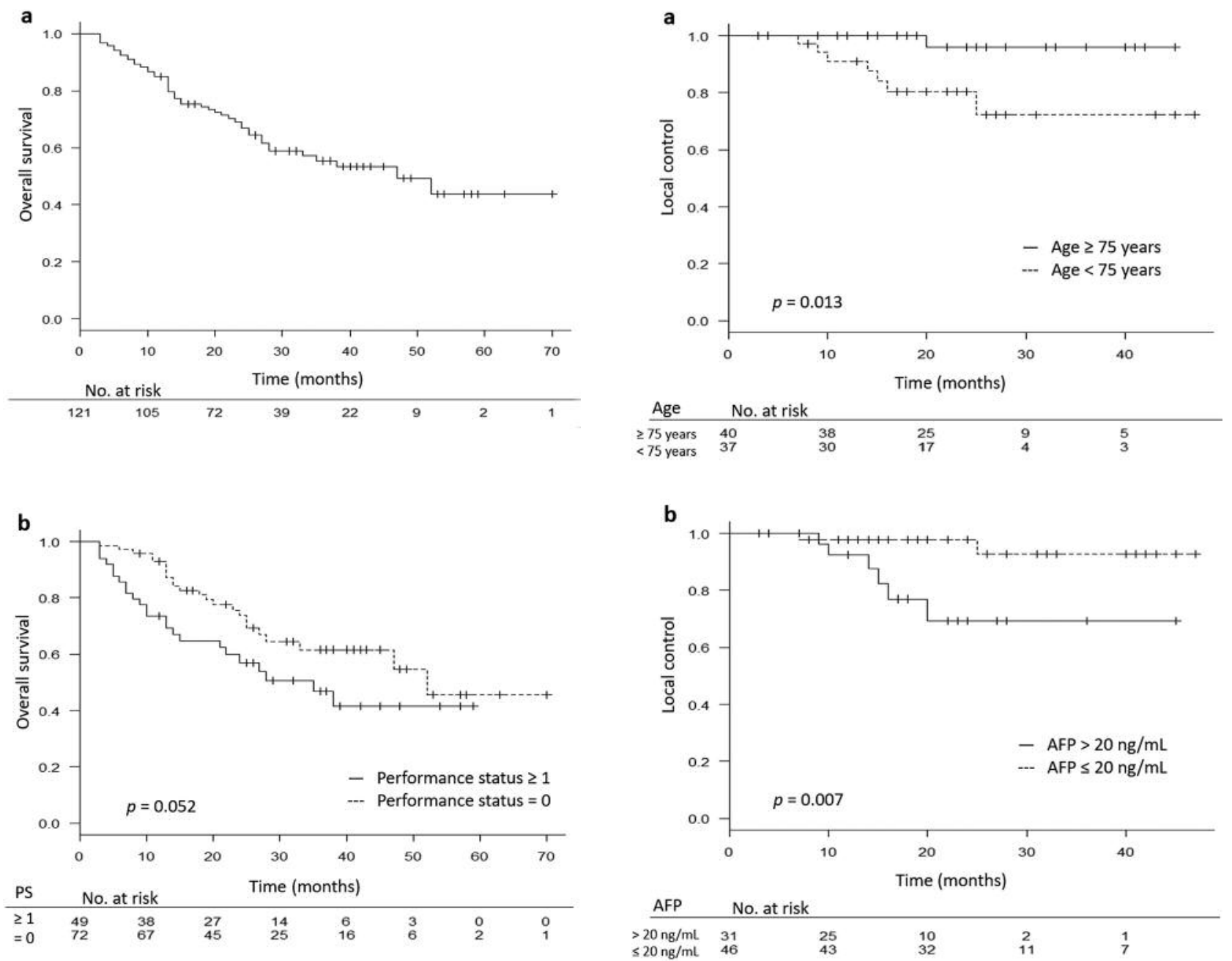

Figure 3. Local control rates in 77 patients treated with a planning target volume margin $<8 \mathrm{~mm}$. The local control rates were calculated from the time of the first stereotactic body radiotherapy treatment

C

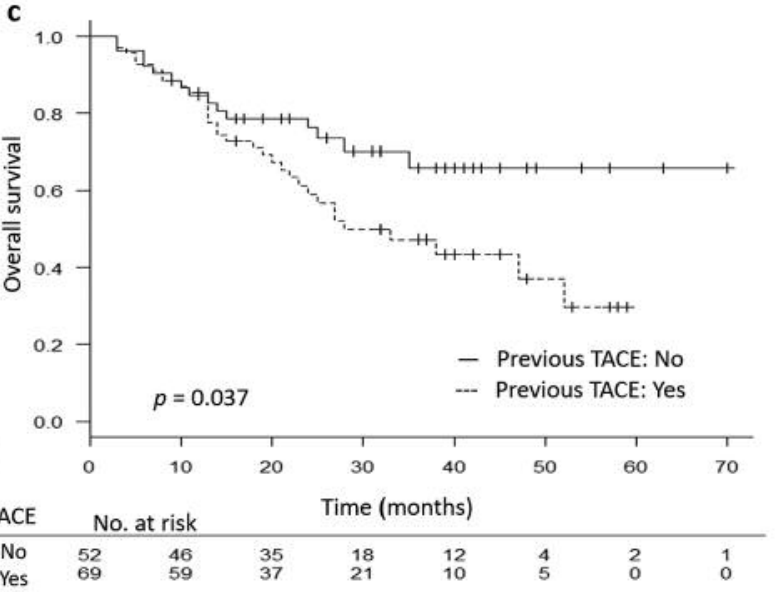
according to age (a) and alpha-fetoprotein (AFP) level (b).

supported by the outcome data in our study, in which a high prevalence of microscopic extension was noted in patients with high AFP values.

This study has some limitations. Firstly, this was a retrospective, single-institution study and not a randomized trial. Therefore, the patients were not controlled with respect to variable prognostic factors. Nevertheless, all patients met the inclusion criteria of prospective studies and received consistent SBRT treatment, which allows for selection bias to be controlled to a considerable degree. In addition, we analyzed a relatively large number of patients with a reliable follow-up period. Moreover, to the best of our knowledge, this is the first report of the possible requirements for CTV margins derived from clinical data after SBRT treatment for

2. Overall survival from the time of the first stereotactic $b$ radiotherapy treatment in all patients $(n=121)(a)$, and according to performance status $(P S)(b)$, and history of previous transarterial chemoembolization (TACE) (c). 
HCC. A second limitation is that the biological characteristics of tumor microinvasion were unclear due to insufficient pathological examination in the present study. A future comparison study between clinical and histological data, using surgically resected specimens, may validate our findings.

In conclusion, in this study, we analyzed the clinical outcomes of patients receiving SBRT for HCC, focusing on the irradiation volume rather than the magnitude of the dose. As a result, it was revealed that the risk of recurrence was increased by reducing the PTV margin and was associated with elevated AFP level. In patients with high AFP levels, there is a possibility of microscopic invasion around the tumor, suggesting that the LC may be improved by adding an additional CTV margin to the GTV.

\section{Ethics approval and consent to participate}

The study design was approved by the Medical Corporation SHINMEIKAI Ethics review board in June 2016 (Approval No. 9) and retrospectively registered.

\section{Conflicts of Interest}

The Authors declare that they have no competing interests in regard to this study.

\section{Acknowledgements}

This work was supported by JSPS KAKENHI Grant Number JP17H04119. The Authors would like to thank Editage (www.editage.jp) for English language editing.

\section{References}

1 Sanyal AJ, Yoon SK and Lencioni R: The etiology of hepatocellular carcinoma and consequences for treatment. Oncologist 15(Suppl 4): 14-22, 2010.

2 Arii S, Yamaoka Y, Futagawa S, Inoue K, Kobayashi K, Kojiro M, Makuuchi M, Nakamura Y, Okita K and Yamada R: Result of surgical and nonsurgical treatment for small-sized hepatocellular carcinomas: a retrospective and nationwide survey in Japan. The Liver Cancer Study Group of Japan. Hepatology 32: 1224-1229, 2000.

3 Ryu M, Shimamura Y, Kinoshita T, Konishi M, Kawano N, Iwasaki M, Furuse J, Yoshino M, Moriyama N and Sugita M: Therapeutic results of resection, transcatheter arterial embolization and percutaneous transhepatic ethanol injection in 3225 patients with hepatocellular carcinoma: a retrospective multicenter study. Jpn J Clin Oncol 27: 251-257, 1997.

4 Blomgren H, Lax I, Näslund I and Svanström R: Stereotactic high-dose fraction radiation therapy of extracranial tumors using an accelerator: Clinical experience of the first thirty-one patients. Acta Oncol 34: 861-870, 1995.

5 Seong J, Park HC, Han KH and Chon Y: Clinical results and prognostic factors in radiotherapy for unresectable hepatocellular carcinoma: a retrospective study of 158 patients. Int $\mathrm{J}$ Radiat Oncol Biol Phys 55: 329-336, 2003.
6 Rosu M, Dawson LA, Balter JM, McShan DL, Lawrence TS and Ten Haken RK: Alterations in normal liver doses due to organ motion. Int J Radiat Oncol Biol Phys 57: 1472-1479, 2003.

7 Choi BO, Jang HS, Kang KM, Lee SW, Kang YN, Chai GY and Choi IB: Fractionated stereotactic radiotherapy in patients with primary hepatocellular carcinoma. Jpn J Clin Oncol 36: 154-158, 2006.

8 Kim TH, Kim DY, Park JW, Kim YI, Kim SH, Park HS, Lee WJ, Park SJ, Hong EK and Kim CM: Three-dimensional conformal radiotherapy of unresectable hepatocellular carcinoma patients for whom transcatheter arterial chemoembolization was ineffective or unsuitable. Am J Clin Oncol 29: 568-575, 2006.

9 Kim Y II, Park HC, Lim DH, Park HJ, Kang SW, Park SY, Kim JS, Han Y and Paik SW: Change of the liver volume and ChildPugh score after high dose hypofractionated radiotherapy in patients with small hepatocellular carcinoma. Radiat Oncol J 30: 189-196, 2012.

10 Wang PM, Chung NN, Hsu WC, Chang FL, Jang CJ and Scorsetti M: Stereotactic body radiation therapy in hepatocellular carcinoma: Optimal treatment strategies based on liver segmentation and functional hepatic reserve. Rep Pract Oncol Radiother 20: 417-424, 2015.

11 Kwon JH, Bae SH, Kim JY, Choi BO, Jang HS, Jang JW, Choi JY, Yoon SK and Chung KW: Long-term effect of stereotactic body radiation therapy for primary hepatocellular carcinoma ineligible for local ablation therapy or surgical resection. Stereotactic radiotherapy for liver cancer. BMC Cancer 10: 475, 2010.

12 Seo YS, Kim MS, Yoo SY, Cho CK, Choi CW, Kim JH, Han CJ, Park SC, Lee BH, Kim YH and Lee DH: Preliminary result of stereotactic body radiotherapy as a local salvage treatment for inoperable hepatocellular carcinoma. J Surg Oncol 102: 209-214, 2010.

13 Andolino DL, Johnson CS, Maluccio M, Kwo P, Tector AJ, Zook J, Johnstone PA and Cardenes HR: Stereotactic body radiotherapy for primary hepatocellular carcinoma. Int J Radiat Oncol Biol Phys 81: e447-e453, 2011.

14 Huang WY, Jen YM, Lee MS, Chang LP, Chen CM, Ko KH, Lin KT, Lin JC, Chao HL, Lin CS, Su YF, Fan CY and Chang YW: Stereotactic body radiation therapy in recurrent hepatocellular carcinoma. Int J Radiat Oncol Biol Phys 84: 355-361, 2012.

15 Honda Y, Kimura T, Akita H, Kobayashi T, Fukuhara T, Masaki K, Nakahara T, Naeshiro N, Ono A, Miyaki D, Nagaoki Y, Kawaoka T, Takaki S, Hiramatsu A, Ishikawa M, Kakizawa H, Kenjo M, Takahashi S, Awai K, Nagata Y and Chayama K: Stereotactic body radiation therapy combined with transcatheter arterial chemoembolization for small hepatocellular carcinoma. J Gastroenterol Hepatol 28: 530-536, 2013.

16 Bujold A, Massey CA, Kim JJ, Brierley J, Cho C, Wong RK, Dinniwell RE, Kassam Z, Ringash J, Cummings B, Sykes J, Sherman M, Knox JJ and Dawson LA: Sequential phase I and II trials of stereotactic body radiotherapy for locally advanced hepatocellular carcinoma. J Clin Oncol 31: 1631-1639, 2013.

17 Jang WI, Kim MS, Bae SH, Cho CK, Yoo HJ, Seo YS, Kang JK, Kim SY, Lee DH, Han CJ, Kim J, Park SC, Kim SB, Cho EH and Kim YH: High-dose stereotactic body radiotherapy correlates increased local control and overall survival in patients with inoperable hepatocellular carcinoma. Radiat Oncol 8: 250, 2013. 
18 Takeda A, Sanuki N, Eriguchi T, Kobayashi T, Iwabutchi S, Matsunaga K, Mizuno T, Yashiro K, Nisimura S and Kunieda E: Stereotactic ablative body radiotherapy for previously untreated solitary hepatocellular carcinoma. J Gastroenterol Hepatol 29: 372-379, 2014.

19 Huertas A, Baumann AS, Saunier-Kubs F, Salleron J, Oldrini G, Croisé-Laurent V, Barraud H, Ayav A, Bronowicki JP and Peiffert D: Stereotactic body radiation therapy as an ablative treatment for inoperable hepatocellular carcinoma. Radiother Oncol 115: 211-216, 2015.

20 Su TS, Lu HZ, Cheng T, Zhou Y, Huang Y, Gao YC, Tang MY, Jiang HY, Lian ZP, Hou EC and Liang P: Long-term survival analysis in combined transarterial embolization and stereotactic body radiation therapy versus stereotactic body radiation monotherapy for unresectable hepatocellular carcinoma $>5 \mathrm{~cm}$. BMC Cancer 16: 834, 2016.

21 Wahl DR, Stenmark MH, Tao Y, Pollom EL, Caoili EM, Lawrence TS, Schipper MJ and Feng M: Outcomes after stereotactic body radiotherapy or radiofrequency ablation for hepatocellular carcinoma. J Clin Oncol 34: 452-459, 2016.

22 Engels B, Soete G, Verellen D and Storme G: Conformal arc radiotherapy for prostate cancer: Increased biochemical failure in patients with distended rectum on the planning computed tomogram despite image guidance by implanted markers. Int $\mathbf{J}$ Radiat Oncol Biol Phys 74: 388-391, 2009.

23 Nagata Y: Stereotactic Body Radiation Therapy: Principles and Practices, First Edition. Tokyo: Springer, 2015.

24 Doi H, Shiomi H, Masai N, Tatsumi D, Igura T, Imai Y and Oh RJ: Threshold doses and prediction of visually apparent liver dysfunction after stereotactic body radiation therapy in cirrhotic and normal livers using magnetic resonance imaging. J Radiat Res 57: 294-300, 2016.

25 National Cancer Institute: Common Terminology Criteria for Adverse Events version 3.0 (CTCAE). Available at https://ctep.cancer.gov/protocoldevelopment/

26 Jwo SC, Chu JH, Chau GY, Loong CC and Lui WY: Risk factors linked to tumor recurrence of human hepatocellular carcinoma after hepatic resection. Hepatology 16: 1367-1371, 1992.

27 Wang W, Feng X, Zhang T, Jin J, Wang S, Liu Y, Song Y, Liu $\mathrm{X}, \mathrm{Yu} \mathrm{Z}$ and $\mathrm{Li} \mathrm{Y}$ : Prospective evaluation of microscopic extension using whole-mount preparation in patients with hepatocellular carcinoma: Definition of clinical target volume for radiotherapy. Radiat Oncol 5: 73, 2010.

28 Wang MH, Ji Y, Zeng ZC, Tang ZY, Fan J, Zhou J, Zeng MS, $\mathrm{Bi}$ AH and Tan YS: Impact factors for microinvasion in patients with hepatocellular carcinoma: possible application to the definition of clinical tumor volume. Int J Radiat Oncol Biol Phys 76: 467-476, 2010.
29 The Cancer of the Liver Italian Program (CLIP) Investigators: A new prognostic system for hepatocellular carcinoma: A retrospective study of 435 patients. Hepatology 28: 751-755, 1998.

30 Chevret S, Trinchet JC, Mathieu D, Rached AA, Beaugrand M and Chastang C: A new prognostic classification for predicting survival in patients with hepatocellular carcinoma. J Hepatol 31: 133-141, 1999.

31 Tangkijvanich P, Anukulkarnkusol N, Suwangool P, Lertmaharit S, Hanvivatvong O, Kullavanijaya P and Poovorawan Y: Clinical characteristics and prognosis of hepatocellular carcinoma: analysis based on serum alpha-fetoprotein levels. J Clin Gastroenterol 31: 302-308, 2000.

32 Chan SL, Mo FK, Johnson PJ, Hui EP, Ma BB, Ho WM, Lam KC, Chan AT, Mok TS and Yeo W: New utility of an old marker: serial $\alpha$-fetoprotein measurement in predicting radiologic response and survival of patients with hepatocellular carcinoma undergoing systemic chemotherapy. J Clin Oncol 27: 446-452, 2009.

33 Ikai I, Arii S, Kojiro M, Ichida T, Makuuchi M, Matsuyama Y, Nakanuma Y, Okita K, Omata M, Takayasu K and Yamaoka Y: Reevaluation of prognostic factors for survival after liver resection in patients with hepatocellular carcinoma in a Japanese nationwide survey. Cancer 101: 796-802, 2004.

34 Imamura H, Matsuyama Y, Tanaka E, Ohkubo T, Hasegawa K, Miyagawa S, Sugawara Y, Minagawa M, Takayama T, Kawasaki $\mathrm{S}$ and Makuuchi M: Risk factors contributing to early and late phase intrahepatic recurrence of hepatocellular carcinoma after hepatectomy. J Hepatol 38: 200-207, 2003.

35 Montorsi M, Santambrogio R, Bianchi P, Donadon M, Moroni E, Spinelli A and Costa M: Survival and recurrences after hepatic resection or radiofrequency for hepatocellular carcinoma in cirrhotic patients: a multivariate analysis. J Gastrointest Surg 9: 62-67, 2005.

36 Changchien CS, Chen CL, Yen YH, Wang JH, Hu TH, Lee CM, Wang CC, Cheng YF, Huang YJ, Lin CY and Lu SN: Analysis of 6381 hepatocellular carcinoma patients in southern Taiwan: prognostic features, treatment outcome, and survival. J Gastroenterol 43: 159-170, 2008.
Received October 26, 2017

Revised November 12, 2017 Accepted November 16, 2017 